International Mathematical Forum, 2, 2007, no. 13, 593 - 617

\title{
INPUT-OUTPUT STABILITY FOR OPTIMAL ESTIMATION PROBLEMS
}

\author{
Angelo Alessandri \\ Department of Production Engineering, Thermoenergetics, \\ and Mathematical Models (DIPTEM) \\ University of Genoa \\ P.le Kennedy, Pad. D - 16129 Genova, Italy \\ alessandri@diptem.unige.it \\ Marcello Sanguineti \\ Department of Communications, Computer, and System Sciences (DIST) \\ University of Genoa \\ Via Opera Pia 13 - 16145 Genova, Italy \\ marcello@dist.unige.it
}

\begin{abstract}
Optimal estimation problems for a class of dynamic systems described by nonlinear differential equations are considered under the effect of disturbances. The estimator is a Luenberger observer that depends on an innovation function to be suitably chosen. The optimality criterion is taken as the norm of the estimation error in a function space and is expressed by means of a cost functional dependent on the innovation function. The well-definiteness of such a functional can be guaranteed via a Lyapunov approach and in terms of input-output stability of mappings between function spaces, where the disturbances are the input and the estimation error is the output. In particular, $\mathcal{L}_{p}$ and Sobolev optimality criteria are adopted. In these cases, relationships between internal (asymptotic and exponential) stability and input-output stability are studied and upper bounds on the estimation error are given. The bounds are illustrated by an example and a converse result is presented. In summary, the paper provides conditions for the well-definiteness of a class of optimal estimation problems and represents a departure point to develop efficient solution methodologies.
\end{abstract}


Mathematics Subject Classification: 46N10, 90B99, 93D05, 93D25, 93E10, 37N35, 37N40, 49N99, 62C99.

Keywords: optimal estimation problems, infinite-dimensional optimization, nonlinear dynamic systems, internal stability, input-output stability.

\section{Introduction}

In a variety of applications, one has at one's disposal some incomplete information about a time-varying vector of parameters, which has to be estimated in the presence of disturbances according to an optimality criterion. This the case, for example, where the parameters are the lengths of the queue messages at the nodes of a communication network, the quantities that can vary in an adaptive model, the parametric faults in fault diagnosis, the levels of the reservoirs in water resource management, etc.

If the time variations in the vector to be estimated and in the available information are modeled as a dynamic system and a measurement channel subject to disturbances, respectively, then the desired properties of the estimate can be obtained by exploiting a system-theoretic approach. In general, it is difficult to determine the estimates of the state variables of a dynamic system when they are not fully measurable and are corrupted by disturbances. In this paper, we address the problem of constructing an estimator by looking at both stability and optimality.

Once the structure of the estimator has been chosen (e.g., the Luenberger observer [11]), the stability of the state estimator is usually considered without disturbances by ensuring the asymptotic convergence of the estimation error to zero. As to the optimality, a possible choice consists in selecting a performance index and minimizing (or maximizing) it to derive the estimates. If one wants to have an estimate expressed as a function of the available information and of the disturbances, then an optimal estimator can be searched for within a set of admissible estimators that belong to a space of functions, whose variables are the information and the disturbances themselves. In general, such a space is infinite-dimensional and depends on the application at hand: e.g., a space of square-integrable estimation functions, of functions with an upper bound on their Lipschitz constants, etc.

The specific choice of the optimality criterion depends on additional requirements for the estimate, such as robustness properties, constraints on the vector to be estimated, and assumptions on the disturbances acting on the estimation process. The $\mathcal{L}_{p}$ norm of the estimation error can be chosen as a performance measure to be minimized. For example, the $\mathcal{L}_{2}$ framework enables one to address the so-called $H_{\infty}$ optimal control. In Statistics, the interest in $\mathcal{L}_{p}$ spaces with $p$ equal to or slightly larger than 1 is justified by the fact 
that such $\mathcal{L}_{p}$ norms are more robust than $\mathcal{L}_{2}$ ones [20]. The choice of an $\mathcal{L}_{p}$ optimality criterion entails a functional (or infinite-dimensional) optimization problem, as the minimization has to be performed in terms of elements of an infinite-dimensional space of functions (representing, as mentioned above, the dependence of the estimation error on the available information and on the disturbances).

In such an optimization context, a basic role is played by the relationship between internal stability and $\mathcal{L}_{p}$ stability. The internal stability of the error in estimating the state of a dynamic system requires that, when no disturbance is present, the estimation error should tend to zero from a nonzero initial value; it can be studied by means of Lyapunov functions. In input/output stability analysis, instead, the input (i.e., the disturbances) and the output (i.e., the estimation error) are considered as signals belonging to a suitable space of functions. Loosely speaking, $\mathcal{L}_{p}$ stability requires that, for an input having a certain bound on its $\mathcal{L}_{p}$ norm, the output is bounded in the same norm and such an upper bound is given by the norm of the disturbances plus an additional constant term that depends on the initial conditions.

In this paper, the aforesaid issues are investigated as follows:

1) we associate with the estimation error dynamics a mapping that assigns to the disturbances (regarded as the input) a corresponding estimation error (considered as the output);

2) we introduce an optimal estimation problem, where the optimality criterion is expressed as the minimization of a norm of the estimation error, and we investigate conditions for the well-definiteness of the associate cost functional;

3) we investigate conditions guaranteeing that a certain type of internal stability implies input-output stability with an upper bound on the norm of the estimation error used as optimality criterion.

To the best of our knowledge, the ideas of looking at the stability of estimators from an input-output point of view and of exploiting stabilty properties in optimal estimation problems are new. The results derived here are related to techniques recently proposed for the solution of a class of optimal estimation problems developed in $[1,2]$.

In the case of linear dynamic systems, well-established relationships between internal and input-output stabilities are available (see, e.g., [9]). Early results [26] on the possibility of finding Lyapunov functions for input-output stable nonlinear systems have been developed in [10], where conditions under which finite-gain stability implies local asymptotic stability and global exponential stability implies finite-gain stability have been provided. In [25], a 
local notion of input-output stability, called "small-signal $\mathcal{L}_{p}$ stability," has been defined, and its relationships with asymptotic stability have been studied. Later on, the concept of small-signal $\mathcal{L}_{p}$ stability has been developed by the notion of "gain over a set," whose connections with local Lyapunov stability have also been analyzed [8]. The notion of $\mathcal{L}_{2}$ gain allows one to resolve both stability and robustness issues, and the connection between $\mathcal{L}_{2}$ gain and passivity provides the basis for suitably dealing with nonlinear systems [22].

A type of input-output stability, called $\mathcal{W}$ stability and based on the use of Sobolev spaces as structured sets of input and output signals, has been proposed in [6], where its relationships with asymptotic stability and $\mathcal{L}_{p}$ stability have been addressed (local $\mathcal{W}$ stability is close to, but not coincident with, the small-signal $\mathcal{L}_{p}$ stability described in [25]).

Here we consider $\mathcal{L}_{p}$ and $\mathcal{W}$ optimality criteria for the optimal estimation problem in the case of a class of continuous-time, nonlinear dynamic systems and a corresponding class of estimators. We investigate conditions guaranteeing that the estimation error belongs to (extended) $\mathcal{L}_{p}, 1 \leq p \leq \infty$, and Sobolev spaces of functions when the disturbances belong to the same space. We give conditions for the input-output stability of the estimation-error mapping with respect to such spaces, and we study the relationships of the inputoutput stability with the internal stability, as well as the connections between the $\mathcal{L}_{p}$ and $\mathcal{W}$ stabilities and the non-divergence of the estimators. We provide upper bounds on the $\mathcal{L}_{p}$ and $\mathcal{W}$ gains of the estimation-error mapping.

The paper is organized as follows. Section 2 gathers the smoothness assumptions on the system and measurement functions, defines the structure of the estimator, describes the functional spaces to which the signals belong, and introduces some concepts of input-output stability. Section 3 states the optimal estimation problem by using the $\mathcal{L}_{p}$ optimality criterion and introduces the role played in its solution by the analysis of the relationships between internal and $\mathcal{L}_{p}$ stabilities. Section 4 contains our results on input-output stability to be used for the $\mathcal{L}_{p}$ optimality criterion, and Section 5 deals with their extensions to the case of a Sobolev optimality criterion. An example concerning a class of nonlinear systems is given in Section 6 , where our theorems are illustrated and a converse result on the relationship between the $\mathcal{L}_{1}$ or $\mathcal{L}_{\infty}$ input-output stability and the exponential stability of the estimation error is reported. Conclusions are drawn in Section 7.

\section{Preliminaries}

A quite general class of autonomous dynamic systems can be represented by

$$
\left\{\begin{array}{l}
\dot{x}=f(t, x, w) \\
y=h(t, x, v)
\end{array}\right.
$$


where, for $t \geq t_{0}, x(t) \in X \subseteq \Re^{n}$ is the state vector, $y(t) \in Y \subseteq \Re^{m}$ is the output vector, $w(t) \in W \subset \Re^{r}$ is the system disturbance, and $v(t) \in V \subset \Re^{s}$ is the measurement disturbance. For every positive integer $n$ and every $a \in \Re^{n}$, $\|a\|$ denotes the Euclidean norm of $a$.

Assumption 2.1 Let $B_{x} \triangleq\{x \in X \mid\|x\|<\bar{x}, \bar{x}>0\}, B_{w} \triangleq\{w \in W \mid$ $\|w\|<\bar{w}, \quad \bar{w}>0\}$, and $B_{v} \triangleq\{v \in V \mid\|v\|<\bar{v}, \bar{v}>0\}$. Then

(i) $f:\left[t_{0}, \infty\right) \times X \times W \rightarrow \Re^{n}$ is piecewise-continuous in $t$ and locally Lipschitz in $x \in B_{x}$, uniformly in $w \in B_{w}$ and $t \in\left[t_{0}, \infty\right)$. Moreover, there exist $L_{f}^{x}, L_{f}^{w} \in \Re^{+}$such that $\left\|f\left(t, x_{1}, w\right)-f\left(t, x_{2}, 0\right)\right\| \leq L_{f}^{x}\left\|x_{1}-x_{2}\right\|+L_{f}^{w}\|w\|$, for all $x_{1}, x_{2} \in B_{x}, w \in B_{w}$, and $t \in\left[t_{0}, \infty\right)$;

(ii) $h:\left[t_{0}, \infty\right) \times X \times V \rightarrow \Re^{m}$ is piecewise-continuous in $t$ and locally Lipschitz in $x \in B_{x}$, uniformly in $v \in B_{v}$ and $t \in\left[t_{0}, \infty\right)$. Moreover, there exist $L_{h}^{x}, L_{h}^{v} \in \Re^{+}$such that $\left\|h\left(t, x_{1}, v\right)-h\left(t, x_{2}, 0\right)\right\| \leq L_{h}^{x} \| x_{1}-$ $x_{2}\left\|+L_{h}^{v}\right\| v \|$, for all $x_{1}, x_{2} \in B_{x}, v \in B_{v}$, and $t \in\left[t_{0}, \infty\right)$.

Assumption 2.1 (i) guarantees the existence and uniqueness of a local solution of the differential equation describing the dynamics of the system (1) [15].

To generate an estimate $\hat{x}(t)$ of $x(t)$, we consider a Luenberger estimator with the structure

$$
\dot{\hat{x}}=f(t, \hat{x}, 0)+g(t, \hat{x}, y-h(t, \hat{x}, 0))
$$

where $\hat{x}(t) \in X \subseteq \Re^{n}, t \geq t_{0}$. The estimator dynamics results from the summation of a prediction term, given by the system dynamics, and an innovation term, represented by the function $g:\left[t_{0}, \infty\right) \times X \times Z \rightarrow \Re^{n}, Z \subseteq \Re^{m}$. In the following, $y-h(t, \hat{x}, 0)$ and $g(\cdot, \cdot, \cdot)$ will be called innovation and innovation function, respectively. Such a function is required to verify the following smoothness assumption.

Assumption 2.2 Let $z \triangleq y-h(t, \hat{x}, 0)$ and $B_{z} \triangleq\{z \in Z \mid\|z\|<\bar{z}, \bar{z}>0\}$. Then $g:\left[t_{0}, \infty\right) \times X \times Z \rightarrow \Re^{n}$ is piecewise-continuous in $t$ and locally Lipschitz in $B_{x} \times B_{z}$, uniformly in $t \in\left[t_{0}, \infty\right)$. More specifically, there exist $L_{g}^{x}, L_{g}^{z} \in \Re^{+}$such that $\left\|g\left(t, x_{1}, z_{1}\right)-g\left(t, x_{2}, z_{2}\right)\right\| \leq L_{g}^{x}\left\|x_{1}-x_{2}\right\|+L_{g}^{z}\left\|z_{1}-z_{2}\right\|$ for all $x_{1}, x_{2} \in B_{x}, z_{1}, z_{2} \in B_{z}$, and $t \in\left[t_{0}, \infty\right)$. Moreover, $g(t, \hat{x}, 0)=0$, $\forall \hat{x} \in B_{x}$.

Assumption 2.2 is a sufficient requirement for having a unique local solution of the differential equation (2) describing the estimator by using standard results on the existence and uniqueness of the solution of a differential equation. 
The condition $g(t, \hat{x}, 0)=0 \forall \hat{x} \in B_{x}$ guarantees that, in the absence of disturbances, if there exists $T \geq t_{0}$ such that $\hat{x}(T)=x(T)$, then $\hat{x}(t)=x(t)$ for every $t \geq T$ [27]. The innovation function has been assumed to be of the abovewritten form for the sake of simplicity, although it can be of a more general type, e.g., $\tilde{g}(t, \hat{x}, h(t, x, v), h(t, \hat{x}, 0))$, where $\tilde{g}(t, \hat{x}, h(t, x, v), h(t, \hat{x}, 0))=0$ if $h(t, x, v)=h(t, \hat{x}, 0)[27]$.

The estimation error $e(t) \triangleq x(t)-\hat{x}(t)$ associated with the estimator (2) can be studied in either the absence or the presence of disturbances affecting the system and measurement equations. In the former case, we refer to the system description

$$
\left\{\begin{array}{l}
\dot{x}=f(t, x, 0) \\
y=h(t, x, 0)
\end{array}\right.
$$

and the corresponding error dynamics is given by

$$
\dot{e}=f(t, x, 0)-f(t, \hat{x}, 0)-g(t, \hat{x}, y-h(t, \hat{x}, 0))
$$

(note that the condition $g(t, \hat{x}, 0)=0$ for every $\hat{x} \in B_{x}$ and every $t \geq t_{0}$ guarantees that $e=0$ is an equilibrium point for (4)). In this case, one considers the estimator (2) for the system (1), and so the dynamics of the estimation error is given by

$$
\dot{e}=f(t, x, w)-f(t, \hat{x}, 0)-g(t, \hat{x}, y-h(t, \hat{x}, v)) .
$$

We recall that the estimator (2) is called (global) asymptotic estimator for the system (3) if the estimation error with the dynamics described by (4) has 0 as a (global) asymptotically stable equilibrium point. It is called (global) exponential estimator if the estimation error with the dynamics described by (4) has 0 as a (global) exponentially stable equilibrium point. Finally, the estimator (2) is a non-divergent estimator for the system (1) if there exist $\bar{e}_{0}, c>0$ such that the estimation error with the dynamics described by (5) satisfies $\|e(t)\| \leq c$ for every $t \geq t_{0}$ if $\left\|e\left(t_{0}\right)\right\| \leq \bar{e}_{0}$. If the estimation error is bounded for every $e\left(t_{0}\right) \in \Re^{n}$, the estimator is said to be globally non-divergent.

In the following, the differential equation (5) is regarded as a dynamic system where the disturbances are the input and the estimation error is the output. This input-output representation is introduced by considering $e(t), w(t)$, and $v(t)$ not just as elements of $\Re^{n}, \Re^{r}$, and $\Re^{s}$, respectively, but as signals belonging to suitable function spaces. As we are interested in the behavior of the estimation error with respect to the disturbances, we are led to regard the disturbances themselves as input signals, and the estimation error as the output. Thus, we associate with the system (5) the estimation-error mapping

$$
\mathcal{E}: \mathcal{I} \times \mathcal{J} \rightarrow \mathcal{O}
$$


where $e=\mathcal{E}(w, v) ; \mathcal{I} \times \mathcal{J}$ and $\mathcal{O}$ are the input and output signal spaces, respectively.

Let us now detail the structures of the function spaces that we shall consider as input and output spaces. In the remaining of this section, $s(\cdot)$ denotes a generic signal.

The space $\mathcal{L}_{p}^{n}$, for $p \in[1, \infty)$ and $n \in \mathbb{N}$, is the set of all Lebesguemeasurable functions $s:[0, \infty) \rightarrow \Re^{n}$ such that $\int_{0}^{\infty}\|s(t)\|^{p} d t<\infty$. For every $p \in[1, \infty), \mathcal{L}_{p}^{n}$ is a Banach space with the norm $\|s\|_{p} \triangleq\left(\int_{0}^{\infty}\|s(t)\|^{p} d t\right)^{1 / p} \cdot \mathcal{L}_{2}^{n}$ is a Hilbert space with the inner product defined as $<s_{1}, s_{2}>_{2} \triangleq \int_{0}^{\infty} s_{1}(t)^{\prime} s_{2}(t) d t$ for every $s_{1}, s_{2} \in \mathcal{L}_{2}^{n}$, where, here and in the following, the symbol ' denotes transposition.

The space $\mathcal{L}_{\infty}^{n}$ is the set of all Lebesgue-measurable functions $s:[0, \infty) \rightarrow$ $\Re^{n}$ that are essentially bounded, i.e., such that ess. $\sup _{t>0}\|s(t)\|<\infty$, where "ess. sup" denotes the essential supremum (supremum except on sets of measure zero). $\mathcal{L}_{\infty}^{n}$ is a Banach space with the norm $\|s\|_{\infty} \triangleq$ ess. $\sup _{t \geq 0}\|s(t)\|$. To deal with possibly unbounded signals and unstable systems, the extension of $\mathcal{L}_{p}$ spaces is defined as follows.

The extended space $\mathcal{L}_{p e}^{n}$ is defined as $\mathcal{L}_{p e}^{n} \triangleq\left\{s \mid s_{\tau} \in \mathcal{L}_{p}^{n}, \forall \tau \geq 0\right\}$, where $s_{\tau}(t) \triangleq\left\{\begin{array}{lll}s(t) & , & t \leq \tau \\ 0 & , & t>\tau\end{array}\right.$. We let $<s_{1}, s_{2}>_{2, \tau} \triangleq<s_{1 \tau}, s_{2 \tau}>_{2}$ and $\|s\|_{p, \tau} \triangleq$ $\left\|s_{\tau}\right\|_{p}$ for every $s_{1}, s_{2} \in \mathcal{L}_{2 e}^{n}$ and every $s \in \mathcal{L}_{p e}^{n}, p \in[1, \infty]$. For every $p \in$ $[1, \infty]$ and $n \in \mathbb{N}, \mathcal{L}_{p}^{n}$ is a subset of $\mathcal{L}_{p e}^{n}$. Note that $\mathcal{L}_{p}^{n}$ is a Banach space for every $p \in[1, \infty]$, whereas $\mathcal{L}_{p e}^{n}$ is not; actually, $\left\{\|\cdot\|_{p, \tau}\right\}_{\tau \geq 0}$ is a family of seminorms for $\mathcal{L}_{p e}^{n}$.

\section{The optimal estimation problem with an $\mathcal{L}_{p}$ optimality criterion}

The unknown innovation function $g$ in an estimator with the structure (2) can be determined according to some optimality criterion. As we consider a framework of $\mathcal{L}_{p}$ signals, it is natural to express such a criterion by means of a cost functional given by the $\mathcal{L}_{p}$ norm of a quantity related to the estimation error. Its choice may depend on additional requirements for the estimate, such as robustness properties, constraints on the vector to be estimated, and assumptions on the disturbances. A cost functional well-suited to optimization in an $\mathcal{L}_{p}$ framework is

$$
J_{p, T}=\|e\|_{p, T}
$$

where $p \in[1, \infty]$ and $T>0$.

Let us denote by $\mathcal{G}$ the set of functions that verify Assumption 2.2. Then the following optimal estimation problem can be stated. 
Problem $\mathbf{E}_{\mathcal{L}_{p}}$. For $p \in[1, \infty], x(0)=x_{0}, \hat{x}(0)=\hat{x}_{0}, w \in \mathcal{L}_{p e}^{r}, v \in \mathcal{L}_{p e}^{s}$, and $T>0$, solve

$$
\inf _{g \in \mathcal{G}} J_{p, T}(g),
$$

where $J_{p, T}(g)=\|x-\hat{x}\|_{p, T}, \quad x, \hat{x} \in \mathcal{L}_{p e}^{n}$, and

$$
\left\{\begin{array}{l}
\dot{x}=f(x, w) \\
y=h(x, v) \\
\dot{\hat{x}}=f(\hat{x}, 0)+g(y-h(\hat{x}, 0)) .
\end{array}\right.
$$

Note that, although an instance of Problem $\mathrm{E}_{\mathcal{L}_{p}}$ depends on $p, T, w(t), v(t)$, $x_{0}$, and $\hat{x}_{0}$, to avoid burdening the notation we do not write such dependences. Note also that for an innovation function $g \in \mathcal{G}, x(0)=x_{0}$, and $\hat{x}(0)=\hat{x}_{0}$, the existence of a unique local solution to the differential equations in (9) is guaranteed by the regularity hypotheses on $f, g$, and $h$ (see, for example, [15, Theorem 2.2]).

Problem $\mathrm{E}_{\mathcal{L}_{p}}$ is a functional (or infinite-dimensional) optimization problem, as the minimization (8) has to be performed with respect to elements of an infinite-dimensional space of functions. In other words, the set of admissible innovation functions has to be considered as a subset of a function space that plays the role of the "ambient" space for Problem $\mathrm{E}_{\mathcal{L}_{p}}$. As $\mathcal{G}$ is a subset of the space $\mathcal{C}\left(Z, \Re^{n}\right)$ of continuous, $n$-valued functions in $Z$ with the supremum norm, a natural choice for the ambient space of Problem $\mathrm{E}_{\mathcal{L}_{p}}$ is $\mathcal{C}\left(Z, \Re^{n}\right)$. This choice was made in [1] to study the properties of a class of optimal estimation problems that generalize the so-called $H_{\infty}$ approach.

Under the very general hypotheses made on the functions $f, g$, and $h$ and on the disturbances $v$ and $w$, finding an analytical solution to Problem $\mathrm{E}_{\mathcal{L}_{p}}$ is a very difficult task. Therefore, in [1] we propose a methodology of approximate solution that reduces the functional optimization Problem $\mathrm{E}_{\mathcal{L}_{p}}$ to a sequence of nonlinear (in general) programming problems.

The need for investigating the relationships between internal and inputoutput stabilities arises from the following:

- the non-divergent behavior of the estimation error in Problem $\mathrm{E}_{\mathcal{L}_{p}}$ can be guaranteed by a Lyapunov-function approach, which is studied in terms of internal stability;

- the optimality criterion (8) makes a concept of stability for mappings among (extended) $\mathcal{L}_{p}$ spaces come into play.

Establishing connections between these two types of stability is the purpose of Section 4. We shall use the following definitions of stability in function spaces of signals. 
Definition 3.1 A mapping $F: \mathcal{L}_{p e}^{m} \rightarrow \mathcal{L}_{q e}^{n}$ is finite-gain $\mathcal{L}$-stable if there exist nonnegative constants $\gamma$ and $\beta$ such that

$$
\|F s\|_{q, \tau} \leq \gamma\|s\|_{p, \tau}+\beta
$$

for every $s \in \mathcal{L}_{p e}^{m}$ and every $\tau \in[0, \infty)$. The smallest $\gamma$ for which there exists $\beta$ such that (10) is satisfied is called $\mathcal{L}$ gain.

If $p=q=\infty$, then the definition of $\mathcal{L}$ stability is the well-known notion of bounded input-bounded output (BIBO) stability for dynamic systems. To deal with input-output relationships defined only for a subset of the input space, the following concept of small-signal stability of a mapping has been defined in $[25]$.

Definition 3.2 A mapping $F: \mathcal{L}_{p e}^{m} \rightarrow \mathcal{L}_{q e}^{n}$ is small-signal finite-gain $\mathcal{L}$ stable if inequality (10) is verified for all $s \in \mathcal{L}_{p e}^{m}$ such that, for every $\tau \in$ $[0, \infty)$, there exists $r \in \Re^{+}$satisfying $\sup _{0 \leq t \leq \tau}\|s(t)\| \leq r$.

Note that for small-signal $\mathcal{L}$ stability, for every $\tau \in[0, \infty)$, the instantaneous values of the input have to satisfy $\|s(t)\| \leq r$ for $0 \leq t \leq \tau$, which corresponds to $\left\|s_{\tau}\right\|_{\infty} \leq r$, but it is not necessary that the input signal should be small in the sense of the $\mathcal{L}_{p}$ norm, $p<\infty$.

Referring to the estimation-error mapping (6) associated with the estimationerror dynamics, we now give a definition of stability for estimators within the framework of (extended) $\mathcal{L}_{p}$ spaces of signals.

Definition 3.3 The estimator (2) is a small-signal finite-gain $\mathcal{L}$-stable estimator for the system (1) if the estimation-error mapping (6) is small-signal finite-gain $\mathcal{L}$-stable.

In the following, results based on Lyapunov theory are presented to cast well-posed optimal state estimation problems in $\mathcal{L}_{p}$ and Sobolev function spaces.

\section{$4 \quad \mathcal{L}_{p}$ stability}

For the sake of notational simplicity and without loss of generality, in the following we let $t_{0}=0$. Let $B_{e} \triangleq\left\{e \in \Re^{n} \mid\|e\|<\bar{e}, \bar{e}>0\right\}$ and $\bar{B}_{e} \triangleq\{e \in$ $\left.\Re^{n} \mid\|e\| \leq \bar{e} \cdot \bar{e}>0\right\}$.

The existence of a Lyapunov function for the error dynamics, together with Assumptions 2.1 and 2.2, guarantee that the estimator (2) is an asymptotic 
estimator for the system (3). For example, using standard results from Lyapunov stability (see, e.g., [15, Theorem 3.8]), it can be proved that, if there exists a Lyapunov function $V:[0, \infty) \times E \rightarrow[0, \infty)$ with $E \supseteq \bar{B}_{e}$, such that

(i) $U_{1}(e) \leq V(t, e) \leq U_{2}(e)$;

(ii) $\dot{V}(t, e)=\frac{\partial V}{\partial t}+\frac{\partial V}{\partial e}[f(t, x, 0)-f(t, \hat{x}, 0)-g(t, \hat{x}, y-h(t, \hat{x}, 0))] \leq$ $-c\|e\|^{l}, l>0$

where $U_{1}($.$) and U_{2}($.$) are continuous and positive definite, (2) is an asymptotic$ estimator for the system (3) for every $e(0) \in \bar{B}_{e} \cap E_{2, \rho}$, where $E_{2, \rho} \triangleq\left\{e \in \Re^{n}\right.$ : $\left.U_{2}(e) \leq \rho\right\}$, and $\rho<\min _{\|e\|=\bar{e}} U_{1}(e)$. If $U_{1}(e)$ is radially unbounded, Assumptions 2.1 and 2.2 are globally verified, and $E=\Re^{n}$, then the estimator is globally asymptotic. The above conditions (i) and (ii) are usually referred to as weak detectability properties (see, e.g., [23]).

Similarly, using [15, Corollary 3.4], one can prove that, if there exists a Lyapunov function $V:[0, \infty) \times E \rightarrow[0, \infty)$ with $E \supseteq \bar{B}_{e}$ and positive constants $l, c_{1}, c_{2}$, and $c_{3}$ such that

(i) $c_{1}\|e\|^{l} \leq V(t, e) \leq c_{2}\|e\|^{l}$

(ii) $\dot{V}(t, e)=\frac{\partial V}{\partial t}+\frac{\partial V}{\partial e}[f(t, x, 0)-f(t, \hat{x}, 0)-g(t, \hat{x}, y-h(t, \hat{x}, 0))]$ $\leq-c_{3}\|e\|^{l}$,

then, for every $\|e(0)\|<\sqrt[l]{\frac{c_{1}}{c_{2}}} \bar{e},(2)$ is an exponential estimator for the system (3). If $E=\Re^{n}$ and Assumptions 2.1 and 2.2 are globally verified, the estimator is globally exponential.

When disturbances affect the dynamic and measurement equations, it is not possible to obtain the convergence of the estimation error to zero, but one can search for conditions guaranteeing the boundedness of the estimation error in some sense, e.g., via the input-output $\mathcal{L}_{p}$ stability of the estimationerror mapping. Let us start with a preliminary result, which, although it follows from basic facts concerning Lyapunov functions, is worth noting as it is introductive to our subsequent analysis.

Proposition 4.1 Suppose that Assumptions 2.1 and 2.2 are verified, and that there exists a Lyapunov function $V:[0, \infty) \times E \rightarrow[0, \infty)$, with $E \supseteq B_{e}$ and positive constants $c_{1}$ and $c_{2}$ such that

$$
\begin{aligned}
& \quad \text { (i) } \dot{V}(t, e)=\frac{\partial V}{\partial t}+\frac{\partial V}{\partial e}[f(t, x, 0)-f(t, \hat{x}, 0)-g(t, \hat{x}, y-h(t, \hat{x}, 0))] \leq \\
& -c_{1}\|e\|^{l}, l>1 \\
& \quad \text { (ii) }\left\|\frac{\partial V}{\partial e}\right\| \leq c_{2}\|e\| .
\end{aligned}
$$

Moreover, suppose that $w \in \mathcal{L}_{\infty}^{r}$ and $v \in \mathcal{L}_{\infty}^{s}$. Then (2) is a non-divergent estimator for the system (1). If $E=\Re^{n}$ and Assumptions 2.1 and 2.2 are 
globally verified, the estimator is globally non-divergent under any initial condition $e(0) \in \Re^{n}$.

Proof: According to the above hypotheses, the evaluation of the derivative of $V(t, e)$ gives

$$
\begin{aligned}
\dot{V}(t, e) & =\frac{\partial V}{\partial t}+\frac{\partial V}{\partial e}[f(t, x, w)-f(t, \hat{x}, 0)-g(t, \hat{x}, h(t, x, v)-h(t, \hat{x}, 0))] \\
& =\frac{\partial V}{\partial t}+\frac{\partial V}{\partial e}[f(t, x, 0)-f(t, \hat{x}, 0)-g(t, \hat{x}, h(t, x, 0)-h(t, \hat{x}, 0))] \\
& +\frac{\partial V}{\partial e}[f(t, x, w)-f(t, x, 0)-g(t, \hat{x}, h(t, x, v)-h(t, \hat{x}, 0)) \\
& +g(t, \hat{x}, h(t, x, 0)-h(t, \hat{x}, 0))] \\
& \leq-c_{1}\|e(t)\|^{l}+c_{2}\|e(t)\|\left(L_{f}^{w}\|w(t)\|+L_{h}^{v} L_{g}^{z}\|v(t)\|\right) .
\end{aligned}
$$

As $w \in \mathcal{L}_{\infty}^{r}, v \in \mathcal{L}_{\infty}^{s}$, there exist $M_{w}$ and $M_{v}$ such that $\|w(t)\| \leq M_{w}$ and $\|v(t)\| \leq M_{v}$ for every $t \geq 0$. Then, for $\|e(t)\|>\tilde{e} \triangleq \sqrt[1-1]{\frac{c_{2}}{c_{1}}\left(L_{f}^{w} M_{w}+L_{h}^{v} L_{g}^{z} M_{v}\right)}$, we have $\dot{V}(t, e)<0$, hence $e \in L_{\infty}^{n}$. In practice, the ball centered in 0 of radius $\tilde{e}$ is an attractive invariant set for the estimation error.

Thus, the estimator (2) is non-divergent if the disturbances belong to $\mathcal{L}_{\infty}$. Let us now consider the $\mathcal{L}_{p}$ stability issue; toward this end, let us suppose that the disturbances belong to some $\mathcal{L}_{p}$ space of signals. The following theorem, proved in [1] (see also [3]), gives conditions guaranteeing that (2) is a smallsignal finite-gain $\mathcal{L}_{p^{-}}$stable estimator.

Theorem 4.2 ([1]) Suppose that Assumptions 2.1 and 2.2 are verified, and that there exists a Lyapunov function $V:[0, \infty) \times E \rightarrow[0, \infty)$, with $E \supseteq B_{e}$, and positive constants $c_{1}, c_{2}, c_{3}$, and $c_{4}$ such that

(i) $c_{1}\|e\|^{2} \leq V(t, e) \leq c_{2}\|e\|^{2}$;

(ii) $\dot{V}(t, e)=\frac{\partial V}{\partial t}+\frac{\partial V}{\partial e}[f(t, x, 0)-f(t, \hat{x}, 0)-g(t, \hat{x}, y-h(t, \hat{x}, 0))] \leq$ $-c_{3}\|e\|^{2}$;

(iii) $\left\|\frac{\partial V}{\partial e}\right\| \leq c_{4}\|e\|$.

Then, for every $e(0)$ such that $\|e(0)\|<\bar{e} \sqrt{\frac{c_{1}}{c_{2}}}$ and for every $w \in \mathcal{L}_{p e}^{r}$ and $v \in \mathcal{L}_{p e}^{s}$ such that, for all $\tau \in[0, \infty)$,

$$
\sup _{0 \leq \sigma \leq \tau}\|w(\sigma)\|<\min \left(\bar{w}, \frac{c_{1} c_{3} \bar{e}}{2 c_{2} c_{4} L_{f}^{w}}\right)
$$

and 


$$
\sup _{0 \leq \sigma \leq \tau}\|v(\sigma)\|<\min \left(\bar{v}, \frac{c_{1} c_{3} \bar{e}}{2 c_{2} c_{4} L_{h}^{v} L_{g}^{z}}\right),
$$

the functional used as the optimality criterion for Problem $E_{\mathcal{L}_{p}}$ is bounded.

More specifically, there exist nonnegative constants $\eta, \lambda$, and $\beta$ for which

$$
\begin{gathered}
J_{p, T}=\|e\|_{p, \tau} \leq \eta\|w\|_{p, \tau}+\lambda\|v\|_{p, \tau}+\beta \\
\text { with } \eta=\frac{c_{2} c_{4} L_{f}^{w}}{c_{1} c_{3}}, \lambda=\frac{c_{2} c_{4} L_{h}^{v} L_{g}^{z}}{c_{1} c_{3}} \text { and } \beta=\sqrt{\frac{c_{1}}{c_{2}}}\|e(0)\| \rho, \text { where } \\
\rho= \begin{cases}1 & p=\infty \\
\left(\frac{2 c_{2}}{c_{3} p}\right)^{1 / p}, & p \in[1, \infty) .\end{cases}
\end{gathered}
$$

From the inspection of the proof of Theorem 4.2, it results that only the following conditions on $f$ and $h$, with respect to the disturbances, and on $g$, with respect to the innovation, are necessary: $\|f(t, x, w)-f(t, x, 0)\| \leq L_{f}^{w}\|w\|$, $\|h(t, x, v)-h(t, x, 0)\| \leq L_{h}^{v}\|v\|$, and $\left\|g\left(t, \hat{x}, z_{1}\right)-g\left(t, \hat{x}, z_{2}\right)\right\| \leq L_{g}^{z}\left\|z_{1}-z_{2}\right\|$. However, to guarantee the existence and uniqueness of local solutions to the equations for the system (3) and the estimator (4), $f$ and $h$ have also to be Lipschitz in $x$, and $g$ has to be Lipschitz in $\hat{x}$. Therefore, for this reason, the hypotheses of Theorem 4.2 include Assumptions 2.1 and 2.2 in their entirety.

According to Theorem 4.2, if suitable Lipschitz conditions are verified by the system, measurement, and innovation nonlinearities, and if the disturbances belong to $\mathcal{L}_{p}$, then, for every $\tau \geq 0$, in a small-signal context the $\mathcal{L}_{p}$ norm of the estimation error $e_{\tau}$ is bounded by a linear combination of the $\mathcal{L}_{p}$ norms of the disturbances $w_{\tau}$ and $v_{\tau}$ with one term due to the initial uncertainty in the state value.

Thus, if the hypotheses of Theorem 4.2 are satisfied, the functional used as the optimality criterion in Problem $\mathrm{E}_{\mathcal{L}_{p}}$ is finite.

From Theorem 4.2, we immediately derive the following corollary.

Corollary 4.3 If Assumptions 2.1 and 2.2 are verified and there exists a Lyapunov function $V:[0, \infty) \times E \rightarrow[0, \infty)$, with $E \supseteq B_{e}$, verifying the same hypotheses as in Theorem 4.2, then, for every $e(0)$ such that $\|e(0)\|<\bar{e} \sqrt{\frac{c_{1}}{c_{2}}}$, the mapping $\mathcal{E}: \mathcal{L}_{p e}^{r} \times \mathcal{L}_{p e}^{s} \rightarrow \mathcal{L}_{p e}^{n}$ associated with the estimation-error dynamics $\dot{e}=f(t, x, w)-f(t, \hat{x}, 0)-g(t, \hat{x}, h(t, \hat{x}, v)-h(t, \hat{x}, 0))$ is small-signal finitegain $\mathcal{L}_{p}$-stable. 
If $w \in \mathcal{L}_{p}^{r}, v \in \mathcal{L}_{p}^{s}, \sup _{\sigma \in[0, \infty)}\|w(\sigma)\|<\min \left(\bar{w}, \frac{c_{1} c_{3} \bar{e}}{2 c_{2} c_{4} L_{f}^{w}}\right)$, and $\sup _{\sigma \in[0, \infty)}\|v(\sigma)\|<$ $\min \left(\bar{v}, \frac{c_{1} c_{3} \bar{e}}{2 c_{2} c_{4} L_{h}^{v} L_{g}^{z}}\right)$, then (12) holds with the subscript $\tau$ dropped. Moreover, if Assumptions 2.1 and 2.2 are globally verified and $E=\Re^{n}$, then (12) holds for every $e(0) \in \Re^{n}, w \in \mathcal{L}_{p e}^{r}, v \in \mathcal{L}_{p e}^{s}$ and the mapping $\mathcal{E}$ is finite-gain $\mathcal{L}_{p}$-stable.

Finally, we give a result on the connections between $\mathcal{L}$-stable and nondivergent estimators.

Proposition 4.4 If $w \in \mathcal{L}_{p}^{r} \cap \mathcal{L}_{q}^{r}$ and $v \in \mathcal{L}_{p}^{s} \cap \mathcal{L}_{q}^{s}$ for some $p, q$ such that $\frac{1}{p}+\frac{1}{q}=1$, then a finite-gain $\mathcal{L}$-stable estimator for the system (1) is a non-divergent estimator for the same system.

Proof: As in the proof of Theorem 5.4, from (5), under Assumptions 2.1 and 2.2 , we have

$$
\|\dot{e}(t)\| \leq\left(L_{f}^{x}+L_{g}^{z} L_{h}^{x}\right)\|e(t)\|+L_{f}^{w}\|w(t)\|+L_{g}^{z} L_{h}^{v}\|v(t)\| .
$$

From this and the hypothesis that the estimator is finite-gain $\mathcal{L}$-stable, we obtain $w \in \mathcal{L}_{p}^{r} \cap \mathcal{L}_{q}^{r}, v \in \mathcal{L}_{p}^{s} \cap \mathcal{L}_{q}^{s} \Longrightarrow e, \dot{e} \in \mathcal{L}_{p}^{n} \cap \mathcal{L}_{q}^{n}$, for every $p, q$ such that $\frac{1}{p}+\frac{1}{q}=1$.

As $e \in \mathcal{L}_{p}^{n}$ and $\dot{e} \in \mathcal{L}_{q}^{n}$, with $\frac{1}{p}+\frac{1}{q}=1$, one has $e^{\prime} \dot{e} \in \mathcal{L}_{1}^{1}$. Since $\frac{d}{d t}\|e\|^{2}=$ $2 e^{\prime} \dot{e} \in \mathcal{L}_{1}^{1}$, it follows ${ }^{1}$ that $\|e\|^{2} \in A C^{1}$ and, by applying to $\int_{0}^{t} e(\tau)^{\prime} \dot{e}(\tau) d \tau$ the formula of integration by parts, one has $2 \int_{0}^{t} e(\tau)^{\prime} \dot{e}(\tau) d \tau=\|e(t)\|^{2}-\|e(0)\|^{2}$. Moreover,

$$
\begin{aligned}
\int_{0}^{t} e(\tau)^{\prime} \dot{e}(\tau) d \tau \leq \int_{0}^{t}\left\|e(\tau)^{\prime} \dot{e}(\tau)\right\| d \tau & \leq\left(\int_{0}^{t}\|e(\tau)\|^{p} d \tau\right)^{\frac{1}{p}}\left(\int_{0}^{t}\|\dot{e}(\tau)\|^{q} d \tau\right)^{\frac{1}{q}} \\
& =\|e\|_{p}\|\dot{e}\|_{q},
\end{aligned}
$$

where $\frac{1}{p}+\frac{1}{q}=1$ and the second inequality is obtained by applying Hölder's inequality. Hence, for every $t \geq 0$,

$$
\|e(t)\|^{2} \leq\|e(0)\|^{2}+2\|e\|_{p}\|\dot{e}\|_{q} .
$$

\footnotetext{
${ }^{1}$ Here and in the following, $A C^{n}$ denotes the set of functions $s:[0, \infty) \rightarrow \Re^{n}$ that are absolutely continuous over $[0, \infty)$, i.e., given any $\varepsilon>0$, there exists $\delta>0$ such that $\sum_{i=1}^{k}\left\|s\left(b_{i}\right)-s\left(a_{i}\right)\right\|<\varepsilon$ for every finite system of pairwise disjoint subintervals $\left(a_{i}, b_{i}\right) \subset$ $[0, \infty), i=1, \ldots, k$, satisfying $\sum_{i=1}^{k}\left(b_{i}-a_{i}\right)<\delta\left[16\right.$, p. 336]. If $s \in A C^{n}$, then, according to Lebesgue's theorem [16, p. 340, Theorem 6], $s$ is differentiable almost everywhere, $\dot{s}$ is integrable over any bounded interval $[a, b] \subset[0, \infty)$, and $\int_{a}^{b} \dot{s}(\tau) d \tau=s(b)-s(a)$.
} 
Thus, $e \in \mathcal{L}_{\infty}^{n}$, if $w \in \mathcal{L}_{p}^{r} \cap \mathcal{L}_{q}^{r}$ and $v \in \mathcal{L}_{p}^{s} \cap \mathcal{L}_{q}^{s}$.

Note that $w \in \mathcal{L}_{p}^{r} \cap \mathcal{L}_{q}^{r}$ and $v \in \mathcal{L}_{p}^{s} \cap \mathcal{L}_{q}^{s}$ imply $w \in \mathcal{L}_{p^{*}}^{r}$ and $v \in \mathcal{L}_{p^{*}}^{s}$ for every $p^{*} \in[p, q]$. Thus, when $p=1$ and $q=\infty$, the hypotheses of Proposition 4.4 imply $w \in \mathcal{L}_{1}^{r} \cap \mathcal{L}_{\infty}^{r}$ and $v \in \mathcal{L}_{1}^{s} \cap \mathcal{L}_{\infty}^{s}$, hence $w \in \mathcal{L}_{p}^{r}$ and $v \in \mathcal{L}_{p}^{s}$ for every $p \in[1, \infty]$.

\section{Sobolev stability}

In this section, we consider an optimality criterion different from the one used in Problem $\mathrm{E}_{\mathcal{L}_{p}}$. We address the case in which one wants to find an estimator that minimizes a Sobolev norm of the estimation error. Thanks to the embedding theorems [7] for Sobolev spaces, their choice in the optimality criterion allows one to model further desired properties of the estimator. In this section, we define an optimal estimation problem with a Sobolev optimality criterion, and we study the relationship between internal stability and input-output stabilities of the Sobolev type.

The Sobolev space $\mathcal{W}_{1,2}^{n}$ is defined as $\mathcal{W}_{1,2}^{n} \triangleq\left\{s:[0, \infty) \rightarrow \Re^{n}: s, \dot{s} \in\right.$ $\left.\mathcal{L}_{2}^{n}\right\}$, where $\dot{s}$ denotes the distributional derivative of $s . \mathcal{W}_{1,2}^{n}$ is a Hilbert space with the inner product defined for every $s_{1}, s_{2} \in \mathcal{W}_{1,2}^{n}$ as $<s_{1}, s_{2}>_{\mathcal{W}}=<$ $s_{1}, s_{2}>_{2}+<\dot{s}_{1}, \dot{s}_{2}>_{2}$. We have $\mathcal{W}_{1,2}^{n} \subset \mathcal{L}_{2}^{n}$ and $\|s\|_{2} \leq\|s\|_{\mathcal{W}}$ for every $s \in \mathcal{W}_{1,2}^{n}$.

We let $\mathcal{W}^{n} \triangleq\left\{s:[0, \infty) \rightarrow \Re^{n}: s \in \mathcal{W}_{1,2}^{n} \bigcap A C_{n}\right\}$ and define its extension as $\mathcal{W}_{e}^{n} \triangleq\left\{s:[0, \infty) \rightarrow \Re^{n}: s \in A C^{n}\right.$ and $\left.s_{\tau},(\dot{s})_{\tau} \in \mathcal{L}_{2}^{n}\right\}$ (these spaces have been described in [6] with the additional condition $s(0)=0$; see also [5]). Note that, as, in general, $s_{\tau}$ is not absolutely continuous, we cannot define $\left\|s_{\tau}\right\|_{\mathcal{W}}$; hence, for every $s, s_{1}, s_{2} \in \mathcal{W}_{e}^{n}$, we let $<s_{1}, s_{2}>_{\mathcal{W}, \tau}=<s_{1}, s_{2}>_{2, \tau}$ $+<\dot{s}_{1}, \dot{s}_{2}>_{2, \tau}$ and $\|s\|_{\mathcal{W}, \tau}=\left(<s, s>_{\mathcal{W}, \tau}\right)^{1 / 2}$.

Then we have the following optimal estimation problem.

Problem $\mathbf{E}_{\mathcal{W}}$. For $p \in[1, \infty], x(0)=x_{0}, \hat{x}(0)=\hat{x}_{0}, w \in \mathcal{L}_{p e}^{r}, v \in \mathcal{L}_{p e}^{s}$, and $T>0$, solve

$$
\inf _{g \in \mathcal{G}} J_{p, T}(g)
$$

where $J_{\mathcal{W}, T}(g)=\|x-\hat{x}\|_{\mathcal{W}, T}, \quad x, \hat{x} \in \mathcal{W}_{e}^{n}$, and

$$
\left\{\begin{array}{l}
\dot{x}=f(x, w) \\
y=h(x, v) \\
\dot{\hat{x}}=f(\hat{x}, 0)+g(y-h(\hat{x}, 0)) .
\end{array}\right.
$$


To establish connections between internal and input/output stabilities in Sobolev spaces, we shall use the following two concepts of stability concerning mappings among (extended) $\mathcal{W}$ spaces.

Definition 5.1 A mapping $F: \mathcal{W}_{e}^{m} \rightarrow \mathcal{W}_{e}^{n}$ is finite-gain $\mathcal{W}$-stable if there exist nonnegative constants $\gamma$ and $\beta$ such that

$$
\|F s\|_{\mathcal{W}, \tau} \leq \gamma\|s\|_{\mathcal{W}, \tau}+\beta
$$

for every $s \in \mathcal{W}_{e}^{m}$ and every $\tau \in[0, \infty)$. The smallest $\gamma$ for which there exists $\beta$ such that (15) is satisfied is called $\mathcal{W}$ gain.

Definition 5.2 A mapping $F: \mathcal{W}_{e}^{m} \rightarrow \mathcal{W}_{e}^{n}$ is locally finite-gain $\mathcal{W}$-stable if inequality (15) is satisfied for every $s \in \mathcal{W}_{e}^{m}$ such that $\|s\|_{\mathcal{W}, \tau} \leq \varepsilon$ for some $\varepsilon \in \Re^{+}$.

Referring to the estimation-error mapping (6) associated with the estimationerror dynamics, we now give a definition of stability for estimators within the framework of $\mathcal{W}$ spaces of signals.

Definition 5.3 The estimator (2) is a locally finite-gain $\mathcal{W}$-stable estimator for the system (1) if the estimation-error mapping (6) is locally finite-gain $\mathcal{W}$-stable.

Since Theorem 4.2 and Corollary 4.3 require not only that the disturbances should belong to $\mathcal{L}_{p e}$ but also that they should have a bounded $\|\cdot\|_{\infty, \tau}$ norm, they establish a small-signal stability result. The reason for introducing the space $\mathcal{W}$ is the possibility of exploiting some of its properties not shared by $\mathcal{L}_{2}$ to obtain a local stability result for the estimation-error mapping. Towards this end, the first step consists in proving the finite-gain, small-signal $\mathcal{W}$ stability of the estimation-error mapping (6), which is analogous (for signals in the space $\mathcal{W})$ to the ones of Theorem 4.2 and Corollary 4.3. The first part of the proof exploits the same machinery as the proof of Theorem 4.2; in addition, the Lipschitz properties of the functions involved and the inequality $\|\cdot\|_{2, \tau} \leq$ $\|\cdot\|_{\mathcal{W}, \tau}$ are used, together with some algebraic manipulations.

Theorem 5.4 Suppose that Assumptions 2.1 and 2.2 are verified. There exists a Lyapunov function $V:[0, \infty) \times E \rightarrow[0, \infty)$, with $E \supseteq B_{e}$, verifying the same hypotheses (i), (ii), and (iii) as in Theorem 4.2. Then, for every $e(0)$ such that $\|e(0)\|<\bar{e} \sqrt{\frac{c_{1}}{c_{2}}}$ and for every $w \in \mathcal{W}_{e}^{r}$ and $v \in \mathcal{W}_{e}^{s}$ such that for all $\tau \in[0, \infty)$, the inequalities

$$
\sup _{0 \leq \sigma \leq \tau}\|w(\sigma)\|<\min \left(\bar{w}, \frac{c_{1} c_{3} \bar{e}}{2 c_{2} c_{4} L_{f}^{w}}\right)
$$


and

$$
\sup _{0 \leq \sigma \leq \tau}\|v(\sigma)\|<\min \left(\bar{v}, \frac{c_{1} c_{3} \bar{e}}{2 c_{2} c_{4} L_{h}^{v} L_{g}^{z}}\right)
$$

hold, then there exist nonnegative constants $a, b$, and $c$ for which

$$
\|e\|_{\mathcal{W}, \tau} \leq a\|w\|_{\mathcal{W}, \tau}+b\|v\|_{\mathcal{W}, \tau}+c
$$

where $a \triangleq \sqrt{3 \delta \eta^{2}+\epsilon}, b \triangleq \sqrt{3 \delta \lambda^{2}+\zeta}, c \triangleq \sqrt{3 \delta} \beta, \delta \triangleq 3\left(L_{f}^{x}+L_{g}^{z} L_{h}^{x}\right)^{2}$, $\epsilon \triangleq 3\left(L_{f}^{w}\right)^{2}, \quad \zeta \triangleq 3\left(L_{h}^{v}\right)^{2}, \eta=\frac{c_{2} c_{4} L_{f}^{w}}{c_{1} c_{3}}, \lambda=\frac{c_{2} c_{4} L_{h}^{v} L_{g}^{z}}{c_{1} c_{3}}$, and $\beta=\sqrt{\frac{c_{1}}{c_{3}}}\|e(0)\|$.

Hence the mapping $\mathcal{E}: \mathcal{W}_{e}^{r} \times \mathcal{W}_{e}^{s} \rightarrow \mathcal{W}_{e}^{n}$ associated with the estimation-error dynamics $\dot{e}=f(t, x, w)-f(t, \hat{x}, 0)-g(t, \hat{x}, h(t, \hat{x}, v)-h(t, \hat{x}, 0))$ is smallsignal finite-gain $\mathcal{W}$-stable.

Proof: As in the proof of Proposition 4.1, the evaluation of the derivative of $V(t, e)$ gives

$$
\dot{V}(t, e) \leq-c_{3}\|e(t)\|^{2}+c_{4}\|e(t)\|\left(L_{f}^{w}\|w(t)\|+L_{h}^{v} L_{g}^{z}\|v(t)\|\right)
$$

Let $W(t) \triangleq \sqrt{V(t, e(t))}$. If $V \neq 0$, from $\dot{W}=\dot{V} / 2 \sqrt{V}$ and hypothesis (i), we derive $\dot{W} \leq-\frac{c_{3}}{2 c_{2}} W(t)+\frac{c_{4}}{2 \sqrt{c_{1}}}\left(L_{f}^{w}\|w(t)\|+L_{h}^{v} L_{g}^{z}\|v(t)\|\right)$. If $V=0$, it is possible to verify that

$$
D^{+} W(t) \leq \frac{c_{4}}{2 \sqrt{c_{1}}}\left(L_{f}^{w}\|w(t)\|+L_{h}^{v} L_{g}^{z}\|v(t)\|\right)
$$

where $D^{+} W(t)$ denotes the upper right-hand derivative of $W(t)^{2}$. Thus, along the lines of [15, Theorem 6.1], we have proved that

$$
D^{+} W(t) \leq-\frac{c_{3}}{2 c_{2}} W(t)+\frac{c_{4}}{2 \sqrt{c_{1}}}\left(L_{f}^{w}\|w(t)\|+L_{h}^{v} L_{g}^{z}\|v(t)\|\right) \text {. }
$$

Then the Comparison Lemma (see, e.g., [15, p. 85]) gives

$$
\begin{aligned}
W(t) & \leq e^{-\frac{c_{3}}{2 c_{2}} t} W(0)+\frac{c_{4}}{2 \sqrt{c_{1}}}\left[L_{f}^{w} \int_{0}^{t} e^{-\frac{c_{3}}{2 c_{2}}(t-\tau)}\|w(\tau)\| d \tau\right. \\
& \left.+L_{h}^{v} L_{g}^{z} \int_{0}^{t} e^{-\frac{c_{3}}{2 c_{2}}(t-\tau)}\|v(\tau)\| d \tau\right]
\end{aligned}
$$

\footnotetext{
${ }^{2}$ We recall that, given $\sigma: \Re \rightarrow \Re$, the upper right-hand derivative $D^{+} \sigma(t)$ is defined as $D^{+} \sigma(t) \triangleq \lim \sup _{\Delta \rightarrow 0^{+}} \frac{\sigma(t+\Delta)-\sigma(t)}{\Delta}$.
} 
Under the hypotheses about the Lyapunov function, the above-written inequality gives

$$
\begin{aligned}
\|e(t)\| \leq \sqrt{\frac{c_{2}}{c_{1}}}\|e(0)\| e^{-\frac{c_{3}}{2 c_{2}} t} & +\frac{c_{4}}{c_{1}}\left[L_{f}^{w} \int_{0}^{t} e^{-\frac{c_{3}}{2 c_{2}}(t-\tau)}\|w(\tau)\| d \tau\right. \\
& \left.+L_{h}^{v} L_{g}^{z} \int_{0}^{t} e^{-\frac{c_{3}}{2 c_{2}}(t-\tau)}\|v(\tau)\| d \tau\right]
\end{aligned}
$$

From (5), under Assumptions 2.1 and 2.2, we have

$$
\begin{aligned}
\|\dot{e}(t)\| & \leq L_{f}^{x}\|x(t)-\hat{x}(t)\|+L_{f}^{w}\|w(t)\|+L_{g}^{z}\left(L_{h}^{x}\|x(t)-\hat{x}(t)\|+L_{h}^{v}\|v(t)\|\right) \\
& =\left(L_{f}^{x}+L_{g}^{z} L_{h}^{x}\right)\|e(t)\|+L_{f}^{w}\|w(t)\|+L_{g}^{z} L_{h}^{v}\|v(t)\| .
\end{aligned}
$$

Thus, we easily obtain ${ }^{3}\|\dot{e}(t)\|^{2} \leq \delta\|e(t)\|^{2}+\epsilon\|w(t)\|^{2}+\zeta\|v(t)\|^{2}$, where $\delta \triangleq 3\left(L_{f}^{x}+L_{g}^{z} L_{h}^{x}\right)^{2}, \epsilon \triangleq 3\left(L_{f}^{w}\right)^{2}$, and $\zeta \triangleq 3\left(L_{g}^{z} L_{h}^{v}\right)^{2}$. Then we have

$$
\|\dot{e}\|_{2, \tau}^{2} \leq \delta\|e\|_{2, \tau}^{2}+\epsilon\|w\|_{2, \tau}^{2}+\zeta\|v\|_{2, \tau}^{2}
$$

Proceeding as in Theorem 4.2 for $p=2$, one can prove the small-signal finitegain $\mathcal{L}_{2}$ stability of the estimation-error mapping, which gives

$$
\|e\|_{2, \tau} \leq \eta\|w\|_{2, \tau}+\lambda\|v\|_{2, \tau}+\beta
$$

for all $\tau \in[0, \infty)$, with $\eta=\frac{c_{2} c_{4} L_{f}^{w}}{c_{1} c_{3}}, \lambda=\frac{c_{2} c_{4} L_{h}^{v} L_{g}^{z}}{c_{1} c_{3}}$, and $\beta=\sqrt{\frac{c_{1}}{c_{3}}}\|e(0)\|$. Now, by using (18) and (19), we obtain

$$
\|\dot{e}\|_{2, \tau}^{2} \leq \delta\left(\eta\|w\|_{2, \tau}+\lambda\|v\|_{2, \tau}+\beta\right)^{2}+\epsilon\|w\|_{2, \tau}^{2}+\zeta\|v\|_{2, \tau}^{2} .
$$

As $\left(\eta\|w\|_{2, \tau}+\lambda\|v\|_{2, \tau}+\beta\right)^{2} \leq 3 \eta^{2}\|w\|_{2, \tau}^{2}+3 \lambda^{2}\|v\|_{2, \tau}^{2}+3 \beta^{2}$, we derive the following upper bound on $\|e\|_{\mathcal{W} \tau}^{2}$ :

$$
\|e\|_{\mathcal{W}, \tau}^{2} \triangleq\|e\|_{2, \tau}^{2}+\|\dot{e}\|_{2, \tau}^{2} \leq\left(3 \delta \eta^{2}+\epsilon\right)\|w\|_{2, \tau}^{2}+\left(3 \delta \lambda^{2}+\zeta\right)\|v\|_{2, \tau}^{2}+3 \delta \beta^{2} .
$$

As $\|w\|_{2, \tau} \leq\|w\|_{\mathcal{W}, \tau}$, and $\|v\|_{2, \tau} \leq\|v\|_{\mathcal{W}, \tau}$, it turns out that

$$
\|e\|_{\mathcal{W}, \tau} \leq \sqrt{3 \delta \eta^{2}+\epsilon}\|w\|_{\mathcal{W}, \tau}+\sqrt{3 \delta \lambda^{2}+\zeta}\|v\|_{\mathcal{W}, \tau}+\sqrt{3 \delta} \beta
$$

\footnotetext{
${ }^{3}$ Here and in the following, we apply the inequality $\left(\sum_{i=1}^{n} a_{i}\right)^{2} \leq n \sum_{i=1}^{n}\left|a_{i}\right|^{2}$ for any $a_{i} \in$ $\Re$.
} 
Remarks analogous to those made after Theorem 4.2 about the Lipschitz conditions on $f, g$, and $h$ can also be made here. Moreover, by analogy to what noted after Corollary 4.3, if $w \in \mathcal{W}^{r}, v \in \mathcal{W}^{s}, \sup _{\sigma \in[0, \infty)}\|w(\sigma)\|<\min$ $\left(\bar{w}, \frac{c_{1} c_{3} \bar{e}}{2 c_{2} c_{4} L_{f}^{w}}\right)$, and $\sup _{\sigma \in[0, \infty)}\|v(\sigma)\|<\min \left(\bar{v}, \frac{c_{1} c_{3} \bar{e}}{2 c_{2} c_{4} L_{h}^{v} L_{g}^{z}}\right)$, then (16) holds with the subscript $\tau$ dropped. Moreover, if Assumptions 2.1 and 2.2 are globally verified and $E=\Re^{n}$, then the result holds for every $e(0) \in \Re^{n}, w \in \mathcal{W}_{e}^{r}, v \in$ $\mathcal{W}_{e}^{s}$, and the mapping $\mathcal{E}$ is finite-gain $\mathcal{W}$-stable.

Theorem 5.4 guarantees that, if suitable Lipschitz conditions are verified by the system, measurement and innovation nonlinearities and if the disturbances belong to $\mathcal{W}_{e}$, then, for any $\tau \geq 0$, in a small-signal context the $\mathcal{W}$ norm of the estimation error $e_{\tau}$ is bounded by a linear combination of the $\mathcal{W}$ norms of the disturbances $w_{\tau}$ and $v_{\tau}$ with one term due to the initial uncertainty in the state value.

Thus, if the hypotheses of Theorem 5.4 are verified, the functional used as the optimality criterion in Problem $\mathrm{E}_{\mathcal{W}}$ is bounded from above by a quantity that can be bounded a priori.

However, Theorem 5.4 in itself does not allow one to conclude that the estimation-error mapping $\mathcal{E}: \mathcal{W}_{e}^{r} \times \mathcal{W}_{e}^{s} \rightarrow \mathcal{W}_{e}^{n}$ is locally $\mathcal{W}$-stable, as the upper bound (16) on the Sobolev norm of the estimation error holds under the hypothesis that the supremum norm of the extended signals is bounded, whereas their Sobolev norm is not so a priori. From now on, we exploit some properties peculiar to the space $\mathcal{W}^{n}$; in this context, such properties make the difference with respect to $\mathcal{L}_{2}$. We recall that $\mathcal{W}^{n}$ is obtained by intersecting the Sobolev space $\mathcal{W}_{1,2}^{n}$ with the space $A C^{n}$ of absolutely continuous functions. As pointed out in [6], signals in $\mathcal{W}$ have the "nice" properties of $\mathcal{L}_{2}$; moreover, they are also in $\mathcal{L}_{\infty}$ and their supremum norm can be bounded in terms of their Sobolev norm. Along the lines of [6], it is just the last property that allows one to prove a local stability result for the estimation-error mapping $\mathcal{E}$. Towards this end, we first present the following lemma, which bounds the supremum norm of signals in $\mathcal{W}^{n}$ in terms of their Sobolev norm and slightly extends [6, Proposition 1].

Lemma 5.5 Let $s \in \mathcal{W}_{e}^{n}$. Then, for all $\tau>0$, we have

$$
\|s\|_{\infty, \tau}^{2} \leq\|s\|_{\mathcal{W}, \tau}^{2}+\|s(0)\|^{2}
$$

Moreover, $\mathcal{W}^{n} \subset \mathcal{L}_{\infty}^{n},\|s\|_{\infty}^{2} \leq\|s\|_{\mathcal{W}}^{2}+\|s(0)\|^{2}$ and $\lim _{t \rightarrow \infty} s(t)=0$

Proof: As $s, \dot{s} \in \mathcal{L}_{2 e}^{n}$, we have $s^{\prime} \dot{s} \in \mathcal{L}_{1 e}^{1}, \frac{d}{d t}\|s\|^{2}=2 s^{\prime} \dot{s} \in \mathcal{L}_{1 e}^{1}$, and then $\left.\|s\|^{2}\right|_{[0, \tau]} \in A C^{1}$ for every $\tau \geq 0$, where $\left.\right|_{[0, \tau]}$ denotes the restriction on the 
interval $[0, \tau]$. (On the other hand, one obtains $\left.\|s\|^{2}\right|_{[0, \tau]} \in A C^{1}$ from the fact that, for any two functions $s_{1}, s_{2} \in A C^{n}$, the function $\tau \longrightarrow s_{1}(\tau)^{\prime} s_{2}(\tau)$ belongs to $\left.A C^{1}\right)$. Then, $2<s, \dot{s}>_{2, t}=\|s(t)\|^{2}-\|s(0)\|^{2}$ for every $t>0$. Now, let $\tau \geq t$; by using Schwarz's inequality, we obtain

$$
\|s(t)\|^{2}-\|s(0)\|^{2} \leq 2\|s\|_{2, t}\|\dot{s}\|_{2, t} \leq\|s\|_{\mathcal{W}, t}^{2} \leq\|s\|_{\mathcal{W}, \tau}^{2} .
$$

Hence $\|s\|_{\infty, \tau}^{2} \leq\|s\|_{\mathcal{W}, \tau}^{2}+\|s(0)\|^{2}$.

If $s \in \mathcal{W}^{n}, \lim _{t \rightarrow \infty} s(t)=0$ according to Barbalat's lemma (for its various versions, see, e.g., [21] and [15, p. 192]), hence $s \in \mathcal{L}_{\infty}^{n}$. Thus, we obtain $\|s\|_{\infty}^{2} \leq\|s\|_{\mathcal{W}}^{2}+\|s(0)\|^{2}$ by taking the limit for $\tau \longrightarrow+\infty$ in $\|s\|_{\infty, \tau}^{2} \leq$ $\|s\|_{\mathcal{W}, \tau}^{2}+\|s(0)\|^{2}$.

Resorting to Lemma 5.5, we are now in a position to prove the following local result.

Corollary 5.6 If Assumptions 2.1 and 2.2 are verified and there exists a Lyapunov function $V:[0, \infty) \times E \rightarrow[0, \infty)$, with $E \supseteq B_{e}$, satisfying the same hypotheses (i), (ii), and (iii) as in Theorem 4.2, then, for every $e(0)$ such that $\|e(0)\|<\bar{e} \sqrt{\frac{c_{1}}{c_{2}}}$, the mapping $\mathcal{E}: \mathcal{W}_{e}^{r} \times \mathcal{W}_{e}^{s} \rightarrow \mathcal{W}_{e}^{n}$ associated with the estimation-error dynamics $\dot{e}=f(t, x, w)-f(t, \hat{x}, 0)-g(t, \hat{x}, h(t, \hat{x}, v)-h(t, \hat{x}, 0))$ is locally $\mathcal{W}$-stable.

Proof: According to Theorem 5.4, there exist $k_{1}, k_{2}, k_{3}>0$ and $\varepsilon_{1}, \varepsilon_{2}>0$ such that $\forall w \in \mathcal{W}_{e}^{r}, \forall v \in \mathcal{W}_{e}^{s}, \forall \tau>0\|w\|_{\infty, \tau}<\varepsilon_{1},\|v\|_{\infty, \tau}<\varepsilon_{2} \Longrightarrow$ $\|e\|_{\mathcal{W}, \tau} \leq k_{1}\|w\|_{\mathcal{W}, \tau}+k_{2}\|v\|_{\mathcal{W}, \tau}+k_{3}$. If we use Lemma 5.5, to guarantee that $\|w\|_{\infty, \tau}<\varepsilon_{1}$ and $\|v\|_{\infty, \tau}<\varepsilon_{2}$, it is sufficient for us to impose $\|w\|_{\mathcal{W}, \tau}<$ $\left(\varepsilon_{1}^{2}-\|w(0)\|^{2}\right)^{1 / 2}$ and $\|v\|_{\mathcal{W}, \tau}<\left(\varepsilon_{2}^{2}-\|v(0)\|^{2}\right)^{1 / 2}$.

\section{An example}

Let us consider the following system, whose dynamics is the summation of a linear term and a nonlinear one, with a linear measurement function:

$$
\left\{\begin{array}{l}
\dot{x}=A x+f(x) \\
y=C x
\end{array}\right.
$$

where, for every $t \geq 0, x(t) \in X \subseteq \Re^{n}$ and $y(t) \in Y \subseteq \Re^{m}$. Let the nonlinearity $f: X \rightarrow \Re^{n}$ be Lipschitz in $B_{x} \triangleq\{x \in X \mid\|x\|<\bar{x}, \bar{x}>0\}$, with the Lipschitz constant $k_{f}$, and suppose that $(A, C)$ is observable. An admissible (in the sense of Assumption 2.2) estimator for such a system is represented by

$$
\dot{\hat{x}}=A \hat{x}+f(\hat{x})+g(y-C \hat{x}),
$$


where $z \triangleq y-C \hat{x} \in Z \subseteq \Re^{m}$ and the innovation function $g: Z \rightarrow \Re^{n}$ is Lipschitz in $B_{z} \triangleq\{z \in Z \mid\|z\|<\bar{z}, \bar{z}>0\}$, and $g(0)=0$. Let the function $g$ be chosen as the summation of two contributions, i.e., $g(y-C \hat{x})=$ $K(y-C \hat{x})+\gamma(y-C \hat{x})$, where $K$ is an $n \times m$ matrix and $\gamma: Z \rightarrow \Re^{n}$ is Lipschitz in $B_{z}$, with the Lipschitz constant $k_{\gamma}$, and $\gamma(0)=0$. Hence the estimator dynamics is given by

$$
\dot{\hat{x}}=A \hat{x}+f(\hat{x})+K(y-C \hat{x})+\gamma(y-C \hat{x}) .
$$

\subsection{Exponential estimator}

First, we study conditions that ensure the asymptotic stability of the estimation error. The dynamics of the estimation error $e(t) \triangleq x(t)-\hat{x}(t)$ is given by

$$
\dot{e}=(A-K C) e+f(x)-f(\hat{x})-\gamma(y-C e) .
$$

Consider the Lyapunov function $V \triangleq e^{\prime} P e$, where the matrix $P$ is positive definite and symmetric; the derivative of $V$ is given by

$$
\begin{aligned}
\dot{V}=e^{\prime}\left[(A-K C)^{\prime} P+P(A-K C)\right] e & +2[f(x)-f(\hat{x})]^{\prime} P e \\
& -2 \gamma^{\prime}(C e) P e .
\end{aligned}
$$

As is usually done in the design of estimators for dynamic systems with Lipschitz nonlinearities $[18,19]$, by using a simple algebra we can compute upper bounds on the last two terms in (23):

$$
\begin{gathered}
2[f(x)-f(\hat{x})]^{\prime} P(x-\hat{x}) \leq 2 k_{f}\|x-\hat{x}\|\|P(x-\hat{x})\| \\
\leq k_{f}^{2}(x-\hat{x})^{\prime} P P(x-\hat{x})+(x-\hat{x})^{\prime}(x-\hat{x})
\end{gathered}
$$

and

$$
\begin{gathered}
-2 \gamma^{\prime}[C(x-\hat{x})] P(x-\hat{x}) \leq 2 k_{\gamma}\|C(x-\hat{x})\|\|P(x-\hat{x})\| \\
\leq k_{\gamma}^{2}\|C\|^{2}(x-\hat{x})^{\prime} P P(x-\hat{x})+(x-\hat{x})^{\prime}(x-\hat{x}) .
\end{gathered}
$$

From (23), by using the above-written inequalities, we obtain

$$
\dot{V} \leq e^{\prime}\left[(A-K C)^{\prime} P+P(A-K C)+\left(k_{f}^{2}+k_{\gamma}^{2}\|C\|^{2}\right) P P+2 I\right] e,
$$

where, given a generic real matrix $R,\|R\| \triangleq \sqrt{\lambda_{\max }\left(R^{\prime} R\right)}$. Given a real symmetric matrix $S, \lambda_{\min }(S)$ and $\lambda_{\max }(S)$ denote the minimum and maximum eigenvalues of $S$, respectively. Note that, for a symmetric matrix $S$, $\|S\|=\left|\lambda_{\max }(S)\right|$. 
Now, if there exist a gain matrix $K$ and a symmetric positive definite matrix $Q$ such that $A-K C$ is Hurwitz and the algebraic Riccati equation

$$
(A-K C)^{\prime} P+P(A-K C)+\left(k_{f}^{2}+k_{\gamma}^{2}\|C\|^{2}\right) P P+2 I=-Q
$$

has a symmetric, positive definite matrix $P$ as its solution, then the estimator (21) admits a quadratic Lyapunov function satisfying the conditions (i) and

(ii) of (11) with $c_{1} \triangleq \lambda_{\text {min }}(P), c_{2} \triangleq \lambda_{\text {max }}(P)$, and $c_{3} \triangleq \lambda_{\text {min }}(Q)$. Hence we conclude that (21) is a global exponential estimator for the system (20). Note that the observability hypothesis about $(A, C)$ is a necessary condition for the existence of such a solution via a suitable choice of the gain $L$.

\subsection{Non-divergent estimator in the presence of additive bounded disturbances}

Suppose that bounded additive disturbances affect the system and measurement equations, that is,

$$
\left\{\begin{array}{l}
\dot{x}=A x+f(x)+w \\
y=C x+v
\end{array}\right.
$$

where $w \in \mathcal{L}_{\infty}^{r}$ and $v \in \mathcal{L}_{\infty}^{s}$, i.e., there exist $M_{w}, M_{v} \in \Re^{+}$such that $\sup _{t \in[0, \infty)}\|w(t)\| \leq M_{w}$ and $\sup _{t \in[0, \infty)}\|v(t)\| \leq M_{v}$. The error dynamics is given by

$$
\dot{e}=(A-K C) e+f(x)-f(\hat{x})+w-K v-\gamma(C e+v) .
$$

If a solution to the algebraic Riccati equation (24) exists, it is easy to verify Proposition 4.1 with $c_{1}=\lambda_{\min }(P)$ and $c_{2}=\lambda_{\max }(P)$, i.e., the fulfilment of the Riccati equation (24) enables one to find a non-divergent estimator in the presence of bounded disturbances.

\section{$6.3 \mathcal{L}_{p}$-stable estimation}

If the algebraic Riccati equation (24) has a symmetric positive definite solution $P$, then Theorems 4.2 and 5.4 can be applied with $L_{f}^{x}=\|A\|+k_{f}, L_{f}^{w}=1$, $L_{g}^{z}=\|K\|+k_{\gamma}, L_{h}^{x}=\|C\|, L_{h}^{v}=1, c_{1}=\lambda_{\min }(P), c_{2}=\lambda_{\max }(P), c_{3}=$ $\lambda_{\min }(Q)$, and $c_{4}=2 \lambda_{\max }(P)$.

More specifically, from Theorem 4.2 we obtain

$$
\|e\|_{p, \tau} \leq \eta\|w\|_{p, \tau}+\lambda\|v\|_{p, \tau}+\beta
$$


for all $\tau \in[0, \infty)$ with $\eta=\frac{2 \lambda_{\max }^{2}(P)\left(\|A\|+k_{f}\right)}{\lambda_{\min }(P) \lambda_{\min }(Q)}, \lambda=\frac{2 \lambda_{\max }^{2}(P)\left(\|K\|+k_{\gamma}\right)}{\lambda_{\min }(P) \lambda_{\min }(Q)}$ and $\beta=\sqrt{\frac{\lambda_{\min }(P)}{\lambda_{\max }(P)}}\|e(0)\| \rho$, where

$$
\rho= \begin{cases}1 & p=\infty \\ \left(\frac{2 \lambda_{\max }(P)}{\lambda_{\min }(Q) p}\right)^{1 / p}, & p \in[1, \infty) .\end{cases}
$$

Analogous calculations can be performed for Theorem 5.4.

\subsection{A converse result}

For nonlinearities $f$ and $\gamma$ with Lipschitz constants satisfying a certain condition, we now derive a converse result according to which the $\mathcal{L}_{1}$ or $\mathcal{L}_{\infty}$ stability of the estimation-error mapping implies the exponential convergence of the estimation error to zero in the absence of disturbances.

Toward this end, consider the error dynamics (26) and assume that $e(0)=$ 0 and that the estimator (21) is $\mathcal{L}_{p}$-stable, for $p=1$ or $p=\infty$. Now, consider the function $u: X \times X \times W \times V \rightarrow \Re^{n}$ defined as $u(x, \hat{x}, w, v) \triangleq$ $f(x)-f(\hat{x})+w-K v-\gamma(C(x-\hat{x})+v)$. Thus, (26) becomes

$$
\dot{e}=(A-K C) e+u \text {. }
$$

By using (27), the smoothness assumptions about $f$ and $\gamma$, and the fact that $e(0)=0$, we obtain

$$
\|u\|_{p} \leq\left[1+\gamma\left(k_{f}+k_{\gamma}\|C\|\right)\right]\|w\|_{p}+\left[\|K\|+\lambda\left(k_{f}+k_{\gamma}\|C\|\right)+k_{\gamma}\right]\|v\|_{p} .
$$

Thus, (28) is an input-output $\mathcal{L}_{p}$-stable linear system for $p=1$ or $p=\infty$, where $u$ is the input and $e$ is the output. We can now apply [24, Theorem 45, p. 301] to conclude that $A-K C$ is Hurwitz. Then, if the disturbances are zero but the initial estimation error $e(0)$ is nonzero, by integrating (22) we obtain

$$
e(t)=\phi(t, 0) e(0)+\int_{0}^{t} \phi(t, \tau)[f(x(\tau))-f(\hat{x}(\tau))-\gamma(C e(\tau))] d \tau,
$$

where $\phi(t, \tau) \triangleq e^{(A-K C)(t-\tau)}$. As $A-K C$ is Hurwitz, there exist $M_{1}, M_{2}>0$ such that $\|\phi(t, \tau)\| \leq M_{1} e^{-M_{2}(t-\tau)}[17$, p. 382]. Thus, we obtain

$$
\|e(t)\| \leq M_{1} e^{-M_{2} t}\|e(0)\|+M_{1} k \int_{0}^{t} e^{-M_{2}(t-\tau)}\|e(\tau)\| d \tau,
$$


where $k \triangleq k_{f}+k_{\gamma}\|C\|$. If we define $\mu(t) \triangleq e^{M_{2} t}\|e(t)\|$, the previous relationship becomes

$$
\mu(t) \leq M_{1} \mu(0)+M_{1} k \int_{0}^{t} \mu(\tau) d \tau .
$$

By applying the Bellman-Gronwall inequality, we obtain

$$
\mu(t) \leq M_{1} \mu(0) e^{M_{1} k t}
$$

and thus

$$
\|e(t)\| \leq M_{1}\|e(0)\| e^{\left(M_{1} k-M_{2}\right) t}
$$

If $M_{1} k-M_{2}<0$, then the same estimator representing an $\mathcal{L}_{1^{-}}$or $\mathcal{L}_{\infty}$-stable estimator is an exponential estimator when no disturbances act on the system and the measurements. In order to guarantee that $e(t)$ lies in the domain of validity of the assumptions, i.e., $e(t) \in B_{e} \triangleq\left\{e \in \Re^{n} \mid\|e\|<\bar{e}, \bar{e}>0\right\}$, $\forall t \geq 0$, it suffices that $\|e(0)\| \leq \frac{\bar{e}}{M_{1}}$.

\section{Conclusions}

In this paper, we have investigated optimal state estimation problems for a class of nonlinear systems by using a Luenberger observer that depends on an innovation function. The novelty with respect to the approaches available in the literature is that the problem is of functional type, where the unknown is the innovation function to be suitably chosen. The optimal estimation problem has been cast in $\mathcal{L}_{p}$ and Sobolev spaces of functions. The well-definiteness of the cost functional to be minimized has been studied in terms of input-output stability of mappings between the input, given by the disturbances, and the output, which is the estimation error.

ACKNOWLEDGMENTS. A. Alessandri and M. Sanguineti were partially supported by a PRIN Grant from the Italian Ministry of University and Research. The authors are grateful to Prof. Saverio Giulini, University of Genoa, for helpful comments.

\section{References}

[1] A. Alessandri, C. Cervellera, and M. Sanguineti, Functional optimal estimation problems and their approximate solution. J. of Optimization Theory and Applications (to appear). 
[2] A. Alessandri, C. Cervellera, and M. Sanguineti, Design of asymptotic state observers for nonlinear systems: an approach based on neural networks and nonlinear programming. IEEE Trans. on Neural Networks (to appear).

[3] A. Alessandri and M. Sanguineti, On estimators for nonlinear systems in $\mathcal{L}_{p}$ spaces, in 39th Conference on Decision and Control, Sydney, Australia, December 2000, pp. 298-303.

[4] A. Alessandri and M. Sanguineti, Approximate solution of optimal estimation problems in $\mathcal{L}_{p}$ spaces, in Proc. NOLCOS'01, Saint Petersburg, Russia, 2001, pp. 866-871.

[5] H. Bourlès, Addendum to W-stability and local input-output stability results, IEEE Trans. on Automatic Control, 45(6) (2000), 1220-1221.

[6] H. Bourlès and F. Colledani, $W$-stability and local input-output stability results, IEEE Trans. on Automatic Control, 40(6) (1995), 1102-1108.

[7] H. Brezis, Analyse Fonctionnelle - Théorie et Applications, Masson, Paris, 1983.

[8] J. Choi, Connections between local stability in Lyapunov and input/output senses, IEEE Trans. on Automatic Control, 40(12) (1995), 2139-2143.

[9] D. F. Delchamps, State Space and Input-Output Linear Systems, SpringerVerlag, New York, 1988.

[10] D. J. Hill and P. J. Moylan, Connections between finite-gain and asymptotic stability, IEEE Trans. on Automatic Control, 25(5) (1980), 931-936.

[11] D. G. Luenberger, Observing the state of a linear system, IEEE Trans. Mil. Electron., 8, (1964), 74-80.

[12] V. Kưrková and M. Sanguineti, Bounds on rates of variable-basis and neural-network approximation, IEEE Trans. on Inform. Theory, 47(6) (2001), 2659-2665.

[13] V. Kůrková and M. Sanguineti, Comparison of worst-case errors in linear and neural network approximation, IEEE Trans. on Inform. Theory, 48(1) (2002), 265-275.

[14] V. Kůrková, M. Sanguineti, Error estimates for approximate optimization by the extended Ritz method. SIAM J. on Optimization, 15 (2005), 461487. 
[15] H. K. Khalil, Nonlinear Systems, Prentice Hall, Upper Saddle River, NJ, 1996.

[16] A. N. Kolmogorov and S. V. Fomin, Introductory Real Analysis, Dover Publications, New York, 1975.

[17] P. Lancaster and M. Tismenetsky, The Theory of Matrices, Academic Press, San Diego, 1985.

[18] S. Raghavan and J. K. Hedrick, Observer design for a class of nonlinear systems, Int. J. of Control, 59(2) (1994), 515-528.

[19] R. Rajamani, Observers for Lipschitz nonlinear systems, IEEE Trans. on Automatic Control, 43(3) (1998), 397-401.

[20] W. J. J. Rey, Introduction to Robust and Quasi-Robust Statistical Methods, Springer-Verlag, Berlin Heidelberg, 1983.

[21] A. R. Teel, Asymptotic convergence from $\mathcal{L}_{p}$ stability, IEEE Trans. on Automatic Control, 44(11) (1999), 2169-2170.

[22] A. van der Shaft, $L_{2}$-Gain and Passivity Techniques in Nonlinear Control, Springer-Verlag, London, 2000.

[23] M. Vidyasagar, On the stabilization of nonlinear systems using state detection, IEEE Trans. on Automatic Control, 25(3) (1980), 504-509.

[24] M. Vidyasagar, Nonlinear Systems Analysis, Prentice Hall, Englewood Cliffs, NJ, 1993.

[25] M. Vidyasagar and A. Vannelli, New relationships between input-output and Lyapunov stability, IEEE Trans. on Automatic Control, 27(2) (1982), 481-483.

[26] J. C. Willems, The generation of Lyapunov functions for input-output stable systems, SIAM J. Contr., 9(1) (1971), 105-133.

[27] X. Xia and W. Gao, On exponential observers for nonlinear systems, Systems $\&$ Control Letters, 11(4) (1988), 319-325.

[28] R. Zoppoli, M. Sanguineti, and T. Parisini, Approximating networks and extended Ritz method for the solution of functional optimization problems, J. of Optimization Theory and Applications, 112(2) (2002), 403440 .

\section{Received: December 20, 2005}

